\title{
Color temperature tunable RGBW clusters with 3 control channels
}

\author{
Daria Kalustova, ${ }^{1}$ Vasyl Kornaga, ${ }^{1}$ Andrii Rybalochka, ${ }^{1}$ Ying-Jie Yu, ${ }^{2}$ Sergiy Valyukh ${ }^{* 3}$ \\ ${ }^{1}$ V. Lashkaryov Institute of Semiconductor Physics NAS of Ukraine, Kyiv, Ukraine, \\ ${ }^{2}$ Department of Precision Mechanical Engineering, Shanghai University, China, \\ ${ }^{3}$ Department of Physics, Chemistry and Biology, Linköping University, Linköping, Sweden
}

Received December 23, 2019; accepted March 27, 2020; published March 31, 2020

\begin{abstract}
The work is devoted to the development of a smart lighting system that is able to change correlated colour temperature and consists of tunable 4-components RGBW clusters controlled via three channels. It is shown that fixing a ratio between the intensities of white and red LEDs at a level of 100:9 enables obtaining high values of colour rendering in a wide range of correlated colour temperatures. The control of 3 from 4 channels simplifies the system. The influence of the red LED on CRI and luminous efficiency is analysed.
\end{abstract}

Light is an important part of human life. Numerous studies on lighting and its influence on our health, as well as the effect on a psychoemotional state and indoor comfort have been done $[1 \div 2]$. Due to the long evolutionary process of human adaptation to natural conditions and the dynamics of lighting changes, natural light is the most comfortable for us. Therefore, an important task in creating light sources is the reproduction of natural daylight parameters, both in terms of the dynamics of intensity and spectral changes providing high color rendering.

The rapid development of solid-state light sources has led to the emergence of functionally new smart lighting systems (SLS). Using several types of LEDs with different spectral power distributions (SPDs), it is possible to get a lighting system being able to change smoothly SPD of the resulting light characterised by correlated colour temperature (CCT), color rendering, and intensity. This enables us to create a comfortable lighting environment.

There are many approaches used for color-tunable LED systems which differ in quantity of LEDs (two and more)

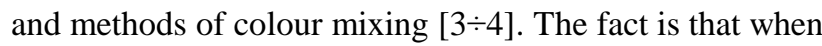
two or three LEDs (control channels) are involved, there is a unique opportunity to determine how LEDs contribute to obtaining the resulting white light. The problem in this case is only choosing the needed LEDs. However, when an SLS includes four and more coloured LEDs, determining the LEDs contribution is not unique. Therefore, further development of algorithms is needed for such a type of SLS requiring additional conditions.

The purpose of this letter is to report on an optimisation method determining the contributions of red, green, blue, and white (RGBW) LEDs in an SLS, which is controlled

\footnotetext{
*E-mail: sergiy.valyukh@liu.se
}

via three channels to achieve high color rendering in a wide CCT range. Colour rendering is analysed in terms of fidelity index $R_{f}$ [5], proposed for scientific application in CIE 224:2017, as well as widely accepted color rendering index (CRI) [6].

In addition to high color rendering at one or another CCT, the advantage of an SLS is its simple controlling principle. There are two alternatives to simplify the control principle of an SLS: reducing the number of different coloured LEDs included in an SLS and optimizing the use of the existing set of LEDs. The first option may have a negative effect on resulting light SPDs or require the use of non-typical phosphor-based LEDs with a wide emission band. Therefore, let us consider the second alternative.

A RGBW SLS consists of three dedicated coloured LEDs and a phosphor-based white (W) LED. Since the CRI values of typical W LEDs are higher than of the white light being generated by three coloured LEDs, it is reasonable to form the resulting light with a significant contribution of the W LED. In this case, the functionality of the coloured LEDs is restricted to the correction of the resulting white light chromaticity coordinates in accordance with CCT.

Analysis of the influence of the W LED on the parameters of the resulting light of a RGBW SLS shows that a warm W LED with a CCT of about $3000 \mathrm{~K}$ and CRI of about 80 is the most effective for obtaining high CRI in a wide CCT range [7]. In order to get the resulting RGBW light having CCT above $3000 \mathrm{~K}$ by using such a W LED, it is necessary to modify its SPD with coloured LEDs, by shifting the chromaticity coordinates in the blue-green region of the CIE diagram. Because of this, the contribution of green (G) and blue (B) LEDs to the resulting spectrum is higher than that of the red (R) LED one. Like in the two white LEDs systems [4], the manipulation of the light spectrum in the RGBW SLS can be done owing to the ratio change between the two parts when the first one contains mostly short wavelengths and the other affects long wavelengths. The ratio between these parts correlates with the contributions of $B$ and $G$ LEDs on the one hand and R and W LEDs on the other 
hand. The study on intensity contributions of $\mathrm{R}$ and $\mathrm{W}$ LEDs to the resulting light showed that these contributions are nearly proportional to each other when it is necessary to obtain high CRI in a wide CCT range. If so, we can determine the optimal ratio between the contributions of $\mathrm{R}$ and $\mathrm{W}$ LEDs, and after that fix their brightnesses ratio and control both LEDs via one channel. This will optimize and simplify the control system of an SLS. Moreover, the fixed ratio of brightness between $\mathrm{R}$ and $\mathrm{W}$ LEDs resolves the problem how to determine the contributions of the B and G LEDs to obtaining a point on the Planck curve. Thus, the further objective of the work is to determine the optimal ratio between the brightnesses of two LEDs (W:R), when the resulting RGBW light has high $\mathrm{R}_{\mathrm{f}}$ and CRI in a wide CCT range.

In order to investigate the problem, two SLSs were studied. They had three identical LEDs: W (2985K, CRI = 82), blue $(461 \mathrm{~nm})$ and green $(525 \mathrm{~nm})$ LEDs, but a different red component. The first one (RGBW SLS) used R LED with a peak wavelength of $625 \mathrm{~nm}$, and the second SLS (dRGBW) used deep red (dR) LED with a peak wavelength of $662 \mathrm{~nm}$.

The SPDs of the resulting light having the chromaticity coordinates coincident with the Planck curve were simulated for different fixed ratios between the brightness of the R and W LEDs for an RGBW SLS, as well as the ratio between the brightness of the $\mathrm{dR}$ and $\mathrm{W}$ LEDs for dRGBW. The resulting light parameters were calculated from these SPDs. The contributions of the $\mathrm{R}$ and $\mathrm{dR}$ LEDs varied from $1 \%$ to $15 \%$ with respect to the contribution of the W LED, the step was $1 \%$. The CCT of the resulting white light therewith changes in the range of $2900 \div 7000 \mathrm{~K}$ with the Duv (Delta u,v) parameter less than 0.0005 . A further increase in $\mathrm{R}$ contribution, as well as that of $\mathrm{dR}$, above $15 \%$ is unreasonable.

The obtained results were analysed in terms of $R_{f}$, CRI and CCT. Some of the curves that enable one to judge the influence of the ratio $\mathrm{R} / \mathrm{W}$, as well as $\mathrm{dR} / \mathrm{W}$, on $R_{f}$ and CRI are presented in Fig. 1 and Fig. 2. The curves are labelled with \% corresponding to the $\mathrm{R}$ and $\mathrm{dR}$ LEDs contributions with respect to the W LED.

According to Fig. 1a, the maximums of $R_{f}$ for different R LED contributions reach $88-94$ in the full CCT range. The obtained dependences show that when the R LED brightness is fixed at a level of $6 \div 9 \%$ of the W LED brightness, the yield $\mathrm{R}_{\mathrm{f}}$ values can be above 90 in the CCT range of $2900 \div 5000 \mathrm{~K}$, but the $9 \%$ of brightness gives higher $\mathrm{R}_{\mathrm{f}}$ values in general. At the same time, for all these R LED brightnesses, the yield CRI values can be above 91 in the full CCT range (Fig. 2). But since most of the daytime natural daylighting CCT is about $5000 \div 6500 \mathrm{~K}$, in terms of maximum CRI values it is reasonable to choose the R LED brightness at the level of $6 \%$ when it http://www.photonics.pl/PLP gives higher CRI values in this CCT range.

a)

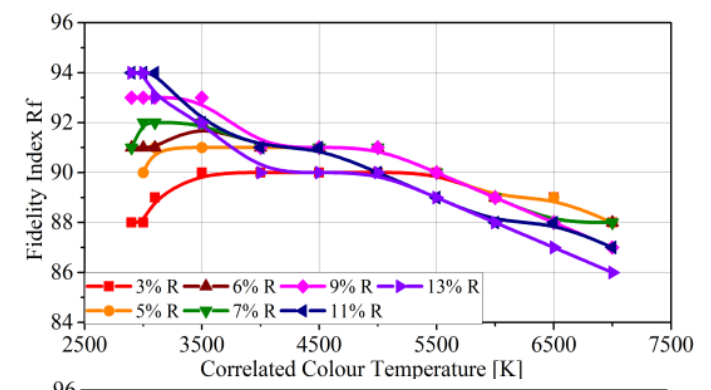

b)

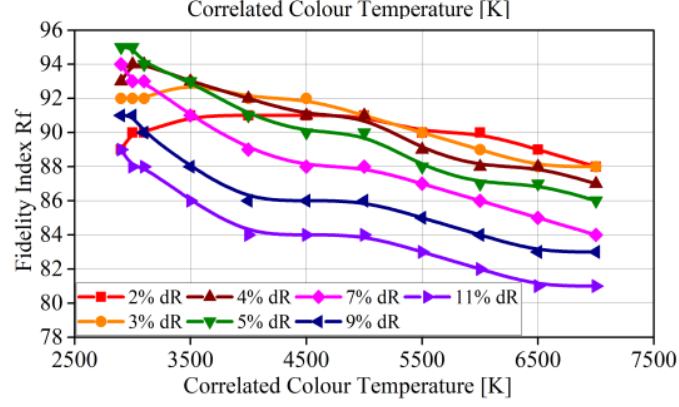

Fig. 1. Fidelity Index $R_{f}$ vs. CCT for RGBW (a) and dRGBW (b) SLSs at different red component contributions.

For the dRGBW SLS, Fig. 1b demonstrates a different curve behaviour compared to the previous case. The contribution of $d R$ LED affects $R_{f}$ and CRI more than that of R LED. A significant decrease is observed of Rf and CRI at high CCTs with an increase in the dR LED brightness contribution from $3 \%$ to $13 \%$. The maximums of Rf reach $88-95$ and the maximums of CRI reach $94-$ 98. Adhering to the similar principle as with the R LED, it is reasonable to fix the dR LED brightness at the level of $3 \%$ of the W LED both in terms of Rf and CRI values. It allows us to obtain the $R_{f}$ values over 90 in the CCT range of $2900 \div 5500 \mathrm{~K}$ and CRI values over 92 in the full CCT range (Fig. 2).

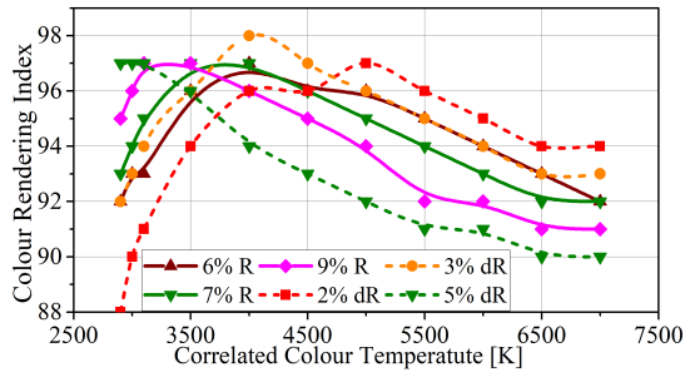

Fig. 2. CRI vs. CCT for RGBW (solid curves) and dRGBW (dashed curves) SLSs at different red component contributions.

Thus, the RGBW lighting system has $R_{f}$ values $87-93$ and CRI values $91-97$ in the CCT range $2900 \div 7000 \mathrm{~K}$ at a fixed ratio R/W equal to 0.09 (9\% of the R LED). A ratio $\mathrm{dR} / \mathrm{W}$ equal to 0.03 (3\% the $\mathrm{dR}$ LED) makes it possible to obtain $R_{f}$ values $88-93$ and CRI values $92-$ 98 in the same CCT range for the dRGBW SLS.

(C) 2020 Photonics Society of Poland 
An additional computation of the gamut index $R_{g}[5 \div 6]$ showed its changing in the ranges from 101 to 102 and from 100 to 101 for the RGBW and dRGBW SLS, respectively. As expected, both SLSs have differences in the values of special colour rendering index R9 (strong red) [6]. The R9 values lay in the range 59-97 and 6395 for the RGBW and dRGBW SLS, respectively.

Besides the colour quality expressed in CRI and $R_{f}$, another important characteristic of a light source is luminous efficiency, $\eta(\mathrm{lm} / \mathrm{W})$. The coloured LEDs considered in the work are typical and widely used. Their luminous efficiencies are $96 \mathrm{~lm} / \mathrm{W}$ for green, $42 \mathrm{~lm} / \mathrm{W}$ for blue, $95 \mathrm{~lm} / \mathrm{W}$ for red, and $25 \mathrm{~lm} / \mathrm{W}$ for deep red (according to the XQ-E series manufactured by Cree Inc). The luminous efficiency of the considered W LED is $180 \mathrm{~lm} / \mathrm{W}$ (according to LG Innotek 5630 series). The obtained dependences of the luminous efficiency on CCT at different red component contributions are shown in Fig. 3. Luminous efficiency decreases with an increase in the $\mathrm{R}$ and $\mathrm{dR}$ LED brightness contribution due to the low $\eta$ of the red component. Luminous efficiencies in the full CCT range change between 109 and $156 \mathrm{~lm} / \mathrm{W}$ for the RGBW SLS at a fixed ratio R/W 0.09, and between $107 \div 148 \mathrm{~lm} / \mathrm{W}$ for dRGBW SLS at a fixed ratio $\mathrm{dR} / \mathrm{W}$ 0.03 . Thus, the difference in luminous efficiencies of the RGBW and dRGBW systems amounts to $2.1 \div 4.6 \%$. The CRI of the white LED increases due to adding coloured $\mathrm{R}, \mathrm{G}, \mathrm{B}$ LEDs by $9 \div 16$ units with a decrease in $\eta$ by $13 \div 40 \%$ depending on the CCT.

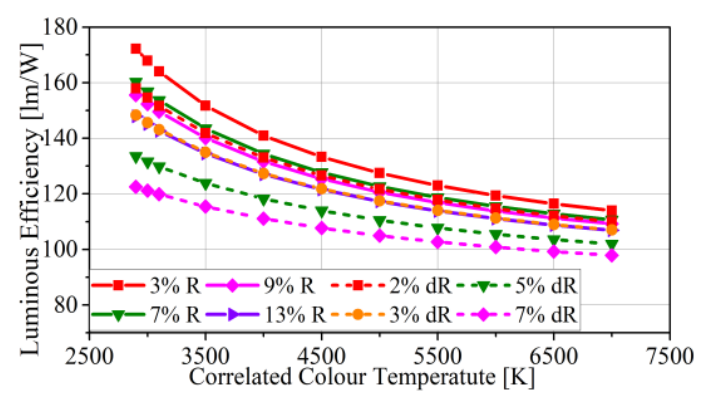

Fig. 3. Luminous efficiency vs. CCT for RGBW (solid curves) and dRGBW (dashed curves) SLSs at different red component contributions

Figure 4 demonstrates the SPDs of the W LED, of the light from the $\mathrm{W}$ and $\mathrm{R}$ LEDs at the R/W ratio 0.09, and the light from the $\mathrm{W}$ and $\mathrm{dR}$ LEDs at the $\mathrm{dR} / \mathrm{W}$ ratio 0.03 .

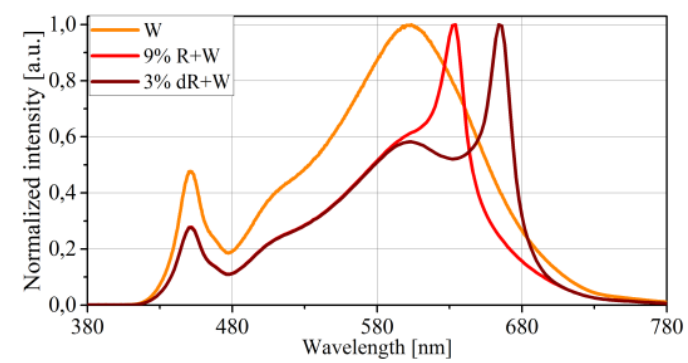

Fig. 4. SPDs of the W LED, and $\mathrm{R}$ and $\mathrm{W}$ LEDs at the R/W ratio 0.09, and $\mathrm{dR}$ and $\mathrm{W}$ LEDs $-0.03(9 \% \mathrm{R}+\mathrm{W}$ and $3 \% \mathrm{dR}+\mathrm{W})$.

http://www.photonics.pl/PLP
According to the study demonstrated above, it is possible to conclude that we can use white LEDs samples that do not conform to the binning system recommended by ANSI C78.377-2008, e.g. white LEDs with chromaticity coordinates being shifted below the Planck curve to the red region of the CIE diagram. This type of LED can be used instead of two LEDs ( $W$ and R), which will simplify the control system and decrease the cost of the system and effectively use a non-standard LED.

In conclusion, we proposed a method for creating a 4component RGBW SLS with three control channels that enables to generate the white light having parameters close to daylight. The simplification of the SLS due to a decrease in the number of controlling channels reduces the cost. This is achieved by fixing the ratio between the brightness of $\mathrm{W}$ and R LEDs, and further control them through one channel. We considered two systems with two different red LEDs. The first system had an ordinary red LED, whereas the second one contained a deep red LED. The study showed that the ratio R/W between the LEDs brightness at a level of 0.09 in the RGBW SLS and the ratio $\mathrm{dR} / \mathrm{W}$ at a level of 0.03 in the dRGBW SLS allow one to obtain the resulting white light in the CCT range of $2900-7000 \mathrm{~K}$ with CRI $91-98$ and $\mathrm{R}_{\mathrm{f}}$ of range from 87 to 93, wherein luminous efficiency $109-$ $156 \mathrm{~lm} / \mathrm{W}$ and $107-148 \mathrm{~lm} / \mathrm{W}$ for these SLS. The achieved luminous efficiencies are about the luminous efficiencies of the existing W LEDs with CRI at a level of 95 and the two LEDs systems (cool W - warm W) with CRI at a level of 90 in a wide CCT range. At the same time, the functionality of the proposed systems is higher.

The proposed method is effective and promising for SLS application and for further studies simplification while maintaining the light quality. However, the aim of the work was not to create a finished product with specific LEDs and electronics, as their choice can affect the parameters of an SLS.

\section{References}

[1] J. Gooley, S. Rajaratnam, G. Brainard, R. Kronauer, C. Czeisler, S. Lockley, Sci Transl. Med. 2, 31 (2010), https://stm.sciencemag.org/ content/2/31/31ra33

[2] A. Borisuit, F. Linhart, J. Scartexxini, M. Munch, Light Res. Technol. 47, 192 (2014), https://journals.sagepub.com/doi/abs/10.1177/ 1477153514531518? journalCode=lrtd

[3] Q. Dai, W. Cai, L. Hao, W Shi, Z. Wang, Light Res. Technol. 50(8), 1198 (2017), https://journals.sagepub.com/doi/10.1177/ 1477153517733504

[4] V. Kornaga, V. Sorokin, A. Rybalochka, O. Oliinyk, N. Kornaga, Semicond. Phys. Quantum. Electron. Optoelectron. 18, 302 (2015), http://journal-spqeo.org.ua/n3_2015/P302-308abstr.html

[5] Illuminating Engineering Society, ANSI/IES TM-30-18 IES method for evaluating light source color rendition (New York, 2018).

[6] Commission International de l'Éclairage, CIE 224:2017 Colour fidelity index for accurate scientific use (Vienna, 2017).

[7] V. Kornaga, D. Kalustova, O. Oliynyk, Int. Sci Conf., Light and power eng: hist, probl, perspect 6, 43 (2018), http://elartu.tntu.edu.ua/ handle/lib/24285?locale $=$ en

(C) 2020 Photonics Society of Poland 